Vietnam Journal of Mechanics, VAST, Vol.43, No. 1 (2021), pp. 43 - 53

DOI: https://doi.org/10.15625/0866-7136/15334

\title{
A MESOSCALE NUMERICAL APPROACH TO PREDICT DAMAGE BEHAVIOR IN CONCRETE BASING ON PHASE FIELD METHOD
}

\author{
Nguyen Hoang Quan ${ }^{1, *}$, Tran Bao Viet ${ }^{1}$, Nguyen Thanh Tung ${ }^{2}$ \\ ${ }^{1}$ Construction Engineering Faculty, Research and Application Center for Technology in Civil \\ Engineering (RACE), University of Transport and Communications, Hanoi, Vietnam \\ ${ }^{2}$ University of Luxembourg, Luxembourg \\ *E-mail: quannh_ktxd@utc.edu.vn
}

Received: 08 August 2020 / Published online: 23 February 2021

\begin{abstract}
In this paper, we develop a numerical approach to simulate the $2 \mathrm{D}$ complex damage and fracture process of quasi-brittle concrete materials. Based on the phase field theory for the case of elastic isotropic multicomponent materials and the generation process based upon Monte Carlo's simulation method, we construct a numerical procedure to solve complex damage thermodynamic problems. The diffusive phase field variable obtained from this calculation can be used to represent the crack nucleation and propagation within 2D complex mesostructure. Some factors that affect the numerical result (type of crack density function and type of split decomposition of strain energy) are accounted to make the predictions more accurate for the case of concrete material. Some new numerical examples are provided to show the usefulness of the approach.
\end{abstract}

Keywords: concrete material, damage, phase field method, Monte Carlo's simulation.

\section{INTRODUCTION}

Over many decades, determining the damage behavior of concrete structure has become a stimulating subject for numerous theoretical-experimental-numerical researches. From the numerical point of view, because of the multiscale characteristic of cementbased materials, a great number of macro-meso-micro simulation approaches exists in the literature to model concrete failure behavior, see for example [1-3] and the references therein. Whereas, the mesoscale approach, that are developed basing on the balance between the microscopic characteristic of material and the global property of concrete structure, takes some advantages to model failure behavior of concrete structure. To date, how to optimize numerical simulation at mesoscale of crack nucleation and propagation in complex brittle composite material like concrete structure is still question in the research domain, see more the review in Introduction of [4]. 
Recently, a numerical smeared crack model named phase field method has been developed. Starting from works of Francfort and Marigo [5], this method aims at constructing a diffusive phase field variable by minimizing the free energy that combines elastic bulk energy and crack surface energy, then this variable can be used to represent the crack network. The phase field method are useful to model crack behavior of brittle material, however application of this method for concrete structure is still need to investigate [6].

Therefore, in this paper, we are interested in construct a simple numerical approximation based on the phase field method within the framework of finite element method to model damage behavior of concrete structure. We present in Section 2 some basics of the phase field method then construct a procedure of discretization of the system of the governing equations at element level using the FEM for displacement and phase field variables. In Section 3, the Monte Carlo simulations are used to construct a generation procedure to generate $2 \mathrm{D}$ concrete structures at mesoscale with randomly circular aggregates of different sizes. In Section 4, the 2D structure are then meshed and the problem are elaborated and calculated in MATLAB for particular examples to show interests of the approximation. Some interesting conclusions will be put in the last section.

\section{THE PHASE FIELD METHOD}

\subsection{Brief review of the phase field method}

In the following, the basic concept of the phase field method is briefly summarized. Consider a cracked body $\Omega \subset \mathbb{R}^{D}$, with $D$ being the space dimension and $\partial \Omega$ its boundary. Let $\Gamma$ be a curve of dimension $D-1$ representing crack discontinuity within $\Omega$. The potential energy of the cracked body in accordance with Griffith's theory can be formulated as follows

$$
E=\int_{\Omega} \psi(\varepsilon) d \Omega+\int_{\Gamma} g_{c} d S
$$

where $\psi$ is the elastic energy density, $\varepsilon$ is the infinitesimal strain tensor which is calculated using small strain theory $\varepsilon=\frac{1}{2}\left(\nabla \mathbf{u}+\nabla \mathbf{u}^{T}\right)$, $\mathbf{u}$ is the vector of displacements, $g_{c}$ is

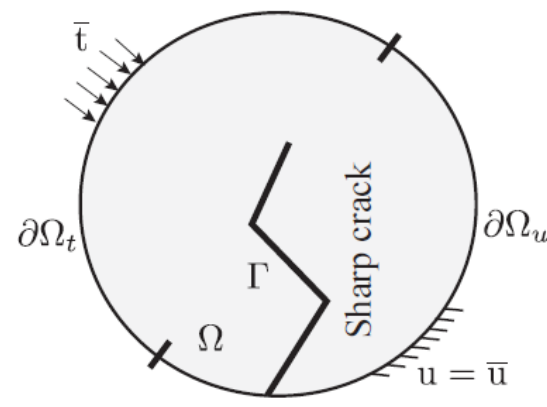

(a) Sharp crack model

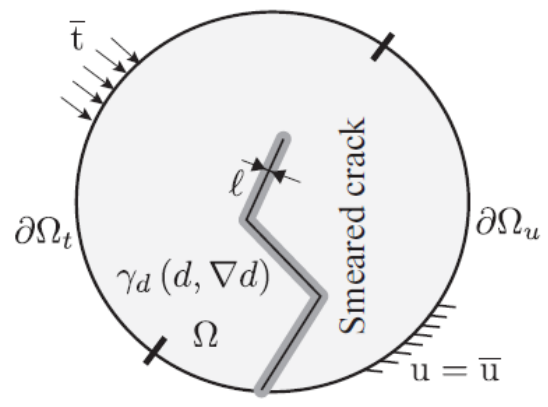

(b) Regularized representation through phase field

Fig. 1. Regularized representation of a crack 
the fracture toughness. In Eq. (1), the first term represents the elastic energy stored in the cracked body and the second stands for the energy required to create the crack according to the Griffith criterion.

In a regularized frame work, the sharp crack is approximated by a smeared representation described by a phase field variable $d(\mathbf{x}) \in[0,1]$. This scalar parameter $d(\mathbf{x})$ takes a unit value on $\Gamma$ and vanished away from it. The phase field indicates the damage level. $d=1$ represents the fully broken state of the material, $d=0$ describes the unbroken state. As a result, the second term in Eq. (1) can be expressed approximately by

$$
\int_{\Gamma} g_{c} d S \simeq \int_{\Omega} g_{c} \gamma_{d}(d, \nabla d) d \Omega,
$$

where $\gamma_{d}(d, \nabla d)$ denotes the crack density function. According to [4,7], it is defined by

$$
\gamma_{d}(d, \nabla d)=\frac{1}{2 l} d^{2}+\frac{l}{2} \nabla d \cdot \nabla d,
$$

$l$ is a regularization parameter related to the width of the smeared crack. A sharp crack is revealed as $l$ approaches the value of zero. It is worth mentioning that, $l$ is also an internal parameter that affects the critical load of crack initiation [8,9]. In order to couple the fracture phase field with the deformation problem, the energy density function can be rewritten as

$$
\psi(\varepsilon, d)=g(d) \psi(\varepsilon),
$$

where $g(d)$ is a parabolic degradation function: $g(d)=(1-d)^{2}+k$ and $k$ is a small numerical parameter to avoid loss of stability in case of fully damaged elements. As a consequence, the total energy in Eq. (1) reads as

$$
E=\int_{\Omega} \psi(\varepsilon, d) d \Omega+\int_{\Omega} g_{c} \gamma_{d}(d, \nabla d) d \Omega
$$

In order to account for a stress degradation only in tension, the elastic strain density is decomposed into positive part due to tension and negative part due to compression

$$
\psi(\varepsilon, d)=g(d) \psi^{+}(\varepsilon)+\psi^{-}(\varepsilon) .
$$

There are several methods which provide the split decomposition of strain energy $[7,9]$. In the present work, the strain decomposition proposed by Miehe et al. [7] is adopted. The elastic strain is decomposed into extensive $\varepsilon^{+}$and compressive $\varepsilon^{-}$parts, with

$$
\varepsilon=\varepsilon^{+}+\varepsilon^{-},
$$

and

$$
\psi^{ \pm}(\varepsilon)=\frac{\lambda}{2}\left[\langle\operatorname{Tr}(\varepsilon)\rangle_{ \pm}\right]^{2}+\mu \operatorname{Tr}\left\{\left(\varepsilon^{ \pm}\right)^{2}\right\}
$$

where

$$
\boldsymbol{\varepsilon}^{+}=\sum_{i=1}^{D}\left\langle\varepsilon^{i}\right\rangle_{+} \mathbf{n}^{i} \otimes \mathbf{n}^{i} \boldsymbol{\varepsilon}^{-}=\sum_{i=1}^{D}\left\langle\varepsilon^{i}\right\rangle-\mathbf{n}^{i} \otimes \mathbf{n}^{i},
$$

where $\varepsilon^{i}$ and $\mathbf{n}^{i}$ are the eigenvalues and eigenvector of $\varepsilon$, i.e satisfying $\boldsymbol{\varepsilon} \mathbf{n}^{i}=\varepsilon^{i} \mathbf{n}^{i}$. In Eq. (9), $\langle x\rangle_{+}=(x+|x|) / 2$ and $\langle x\rangle_{-}=(x-|x|) / 2$. 
Besides, the external energy can be formulated as follows

$$
W^{e x t}=\int_{\Omega} \mathbf{f} \cdot \mathbf{u} d \Omega+\int_{\delta \Omega_{F}} \overline{\mathbf{F}} \cdot \mathbf{u} d \Gamma,
$$

where $\mathbf{f}$ and $\overline{\mathbf{F}}$ are respectively the prescribed volume and boundary force.

The displacement field $\mathbf{u}(\mathbf{x})$ and the phase field $d(\mathbf{x})$ can be determined by applying the principle of maximum dissipation and energy minimization [5]. The problem can be split into two quasi independent minimization procedures. First, with a fixed $\mathbf{u}$, the phase field problem is derived by minimizing the total energy with respect to the phase field

$$
d(\mathbf{x})=\operatorname{Arg}\left\{\inf _{d \in \mathcal{S}_{d}} E(\mathbf{u}, d)\right\}
$$

where $\mathcal{S}_{d}=\{\nabla d \cdot \mathbf{n}=0$ on $\partial \Omega, d \mid \dot{d}(\mathbf{x}) \geq 0,0 \leq d(\mathbf{x}) \leq 1\}$. The evolution law of the phase field to ensure the irreversibility of the process is derived through a thermodynamically consistent framework, see e.g for more details $[4,10,11]$. The weak form of the phase field problem can be written as

$$
\int_{\Omega}\left\{\left(2 \psi^{+}+\frac{g_{c}}{l}\right) d \delta d+g_{c} l \nabla d \cdot \nabla(\delta d)\right\} d \Omega=\int_{\Omega} 2 \psi^{+} \delta d d \Omega .
$$

The history strain energy density function $\mathcal{H}(\mathbf{x}, t)$ is introduced to describe a dependence on history and possible loading-unloading

$$
\mathcal{H}(\mathbf{x}, t)=\max _{\tau \in[0, t]}\left\{\psi^{+}(\mathbf{x}, \tau)\right\} .
$$

The weak form of the phase field problem is finally rewritten as

$$
\int_{\Omega}\left\{\left(2 \mathcal{H}+\frac{g_{c}}{l}\right) d \delta d+g_{c} l \nabla d \cdot \nabla(\delta d)\right\} d \Omega=\int_{\Omega} 2 \mathcal{H} \delta d d \Omega .
$$

Then with a fixed $d$, the mechanical problem consists in minimizing the total energy with respect to displacements:

$$
\mathbf{u}(\mathbf{x})=\operatorname{Arg}\left\{\inf _{u \in \mathcal{S}_{u}}\left(E(\mathbf{u}, d)-W^{\text {ext }}\right)\right\},
$$

where $\mathcal{S}_{u}=\left\{\mathbf{u} \mid \mathbf{u}(\mathbf{x})=\overline{\mathbf{u}}\right.$ on $\left.\partial \Omega_{u}, \mathbf{u} \in H^{1}(\Omega)\right\}$. We obtain the weak form for $\mathbf{u}(\mathbf{x}) \in \mathcal{S}_{u}$ as follows

$$
\int_{\Omega} \sigma: \varepsilon(\delta \mathbf{u}) d \Omega=\int_{\Omega} \mathbf{f} \cdot \delta \mathbf{u} d \Omega+\int_{\partial \Omega_{F}} \overline{\mathbf{F}} \delta \mathbf{u} d \Gamma \forall \delta \mathbf{u} \in H_{0}^{1}(\Omega)
$$

where the Cauchy stress $\sigma=\frac{\partial \psi}{\partial \varepsilon}$ is given by

$$
\boldsymbol{\sigma}=\left((1-d)^{2}+k\right)\left\{\lambda\langle\operatorname{Tr} \varepsilon\rangle_{+} \mathbf{1}+2 \mu \varepsilon^{+}\right\}+\lambda\langle\operatorname{Tr} \varepsilon\rangle_{-} \mathbf{1}+2 \mu \varepsilon^{-} .
$$




\subsection{Finite element discretization}

The problem described in Eqs. (14), (16) are solved by a standard FE procedure in a staggered scheme at each time step, i.e. the phase field problem and the mechanical problem are solved alternatively. For more theoretical and practical details, the interested reader can refer to Miehe et al. [7] and Nguyen et al. [4].

In 2D, the vector form for second order tensor can be expressed as: $[\varepsilon]=\left\{\varepsilon_{11}, \varepsilon_{22}, 2 \varepsilon_{12}\right\}^{T}$ and $[\boldsymbol{\sigma}]=\left\{\sigma_{11}, \sigma_{22}, \sigma_{12}\right\}^{T}$, and $[\mathbf{1}]=\{1,1,0\}^{T}$. The discretization of the system of the governing equations at element level using the FEM for displacement and phase field variables can be expressed as follows

$$
\begin{array}{ll}
\mathbf{u}=\mathbf{N}_{u} \mathbf{u}_{i,} & \mathbf{u}=\mathbf{N}_{u} \delta \mathbf{u}_{i}, \\
{[\mathcal{\varepsilon}(\mathbf{u})]=\mathbf{B}_{u} \mathbf{u}_{i,}} & {[\mathcal{E}(\delta \mathbf{u})]=\mathbf{B}_{u} \delta \mathbf{u}_{i},} \\
d(\mathbf{u})=\mathbf{N}_{d}(\mathbf{x}) \mathbf{d}_{i,} & \nabla d(\mathbf{x})=\mathbf{B}_{d}(\mathbf{x}) \mathbf{d}_{i} \\
\delta d(\mathbf{u})=\mathbf{N}_{d}(\mathbf{x}) \delta \mathbf{d}_{i,}, & \nabla \delta d(\mathbf{x})=\mathbf{B}_{d}(\mathbf{x}) \delta \mathbf{d}_{i},
\end{array}
$$

where $\mathbf{u}_{i}, \mathbf{d}_{i}$ denoting nodal displacements and nodal phase field at time $t_{n+1}$, respectively. $\mathbf{N}_{u, d}, \mathbf{B}_{u, d}$ are vector of shape function and matrix of shape functions derivatives for displacement and phase field, respectively.

The finite element equation of phase field problem is given by

$$
\mathbf{K}_{d} \mathbf{d}_{n+1}=\mathbf{F}_{d}
$$

where

$$
\mathbf{K}_{d}=\int_{\Omega}\left\{\left(\frac{g_{c}}{l}+2 \mathcal{H}_{n}\right) \mathbf{N}_{d}^{T} \mathbf{N}_{d}+g_{c} l \mathbf{B}_{d}^{T} \mathbf{B}_{d}\right\} d \Omega,
$$

and

$$
\mathbf{F}_{d}=\int_{\Omega} 2 \mathbf{N}_{d}^{T} \mathcal{H}_{n} d \Omega,
$$

where $\mathcal{H}_{n}=\mathcal{H}\left(\mathbf{u}_{n}\right)$ is computed from the previous load increment

$$
\left\{\begin{array}{lll}
\mathcal{H}_{n+1}(\mathbf{x})=\psi_{n+1}^{+}(\mathbf{x}) & \text { if } & \psi_{n+1}^{+}(\mathbf{x})>\psi_{n}^{+}(\mathbf{x}) \\
\mathcal{H}_{n+1}(\mathbf{x})=\psi_{n}^{+}(\mathbf{x}) & \text { if } & \psi_{n+1}^{+}(\mathbf{x}) \leq \psi_{n}^{+}(\mathbf{x})
\end{array}\right.
$$

For the mechanical problem, the spectral decomposition of the strain field (Eqs. (7), (8)) cause a strongly nonlinear mechanical problem. To avoid this nonlinearity, the shifted strain tensor split algorithms proposed by the present authors in Ref [4] is adopted. Within the context of incremental scheme, the projection tensors defined at time $n+1$, will be evaluated based on the result from previous time step $n$ as follows

$$
\begin{gathered}
\varepsilon_{n+1}^{ \pm} \simeq \mathbb{P}^{ \pm}\left(\varepsilon_{n}\right): \varepsilon_{n+1}, \\
\left\langle\operatorname{Tr} \varepsilon_{n+1}\right\rangle_{+} \simeq \mathcal{R}^{+}\left(\varepsilon_{n}\right) \operatorname{Tr} \varepsilon_{n+1},\left\langle\operatorname{Tr} \varepsilon_{n+1}\right\rangle_{-} \simeq \mathcal{R}^{-}\left(\varepsilon_{n}\right) \operatorname{Tr} \varepsilon_{n+1},
\end{gathered}
$$


with $\mathcal{R}^{+}\left(\varepsilon_{n}\right)=\frac{1}{2}\left(\operatorname{sign}\left(\operatorname{Tr} \varepsilon_{n}\right)+1\right) ; \mathcal{R}^{-}\left(\varepsilon_{n}\right)=\frac{1}{2}\left(\operatorname{sign}\left(-\operatorname{Tr} \varepsilon_{n}\right)+1\right)$. Setting $\mathcal{R}^{ \pm}\left(\varepsilon_{n}\right) \equiv$ $\mathcal{R}_{n}^{ \pm}, \mathbf{P}^{ \pm}\left(\varepsilon_{n}\right) \equiv \mathbf{P}_{n}^{ \pm}$, where $\mathbf{P}^{ \pm}$are the matrix forms associated with the fourth-order tensors $\mathbb{P}^{ \pm}$. Then the stress at time $t_{n+1}$ can be expressed as

$$
\begin{aligned}
{\left[\sigma_{n+1}\right]=} & \left(\left(1-d_{n+1}\right)^{2}+k\right)\left\{\lambda \mathcal{R}_{n}^{+}\left(\left[\boldsymbol{\varepsilon}_{n+1}\right] \cdot[\mathbf{1}]\right)[\mathbf{1}]+2 \mu \mathbf{P}_{n}^{+}\left[\boldsymbol{\varepsilon}_{n+1}\right]\right\} \\
& +\lambda \mathcal{R}_{n}^{-}\left(\left[\boldsymbol{\varepsilon}_{n+1}\right] \cdot[\mathbf{1}]\right)[\mathbf{1}]+2 \mu \mathbf{P}_{n}^{-}\left[\boldsymbol{\varepsilon}_{n+1}\right] .
\end{aligned}
$$

The finite element equation for the mechanical problem can be written as follows

$$
\left.\left\{\mathbf{K}_{1}\left(d_{n+1}, \mathbf{u}_{n}\right)+\mathbf{K}_{2}\left(\mathbf{u}_{n}\right)\right)\right\} \mathbf{u}_{n+1}=\mathbf{F}_{n+1},
$$

where

$$
\begin{gathered}
\mathbf{K}_{1}\left(d_{n+1}\right)=\int_{\Omega} \mathbf{B}_{u}^{T}\left\{\left(\left(1-d_{n+1}\right)^{2}+k\right)\left(\lambda \mathcal{R}_{n}^{+}[\mathbf{1}]^{T}[\mathbf{1}]+2 \mu \mathbf{P}_{n}^{+}\right)\right\} \mathbf{B}_{u} d \Omega, \\
\mathbf{K}_{2}=\int_{\Omega} \mathbf{B}_{u}^{T}\left\{\lambda \mathcal{R}_{n}^{+}[\mathbf{1}]^{T}[\mathbf{1}]+2 \mu \mathbf{P}_{n}^{+}\right\} \mathbf{B}_{u} d \Omega, \\
\mathbf{F}_{n+1}=\int_{\Omega} \mathbf{N}_{u}^{T} \mathbf{f} d \Omega+\int_{\partial \Omega_{F}} \mathbf{N}_{u}^{T} \overline{\mathbf{F}} d \Gamma .
\end{gathered}
$$

\section{ARRANGEMENT OF AGGREGATE PARTICLE BASED ON MONTE CARLO SIMULATION}

At mesoscopic level, concrete could be represented as biphasic material: coarse aggregates and mortar matrix and an interfacial transition zone (ITZ) between them. The evaluation of the composite behavior of concrete at mesoscopic level requires the generation of a random aggregate structure in which the shape, size and distribution of coarse aggregate closely resemble real concrete in the statistical sense. The shape of aggregate particles depends on the aggregate types. In general, gravel aggregates have a rounded shape while crushed stone aggregates have an angular shape. In 2D numerical simulation, the aggregate shape could be simulated by a polygonal shape [12] and elliptical or circular shape [13].

The size distribution of concrete may be constructed based on an experimental sieving process. Alternatively, the grading of aggregate particle is designed by the Fuller curve which give an optimal density and strength of concrete mixture. The Fuller curve can be expressed as follows

$$
P(d)=100\left(\frac{d}{d_{\max }}\right)^{n},
$$

where $P(d)$ is the cumulative percentage passing a sieve with aperture diameter $d . d_{\max }$ is the and maximum size of aggregate and $n$ is the exponent of the equation. Thus, for an interval $\left[d_{i}, d_{i+1}\right]$ defined by two sequential sieve opening sizes, $d_{i}$ and $d_{i+1}$, then the area of aggregates within a grading segment $\left[d_{i}, d_{i+1}\right]$ can be calculated as:

$$
A_{a g g}\left[d_{i+1}-d_{i}\right]=\frac{P\left(d_{i+1}\right)-P\left(d_{i}\right)}{P\left(d_{\max }\right)-P\left(d_{\min }\right)} P_{a g g} A,
$$


where $A_{\text {agg }}\left[d_{i+1}-d_{i}\right]$ is the area of aggregate within the grading segment $\left[d_{i}, d_{i+1}\right] . d_{\text {min }}$ is the minimum size of aggregate, $P_{a g g}$ is the area fraction of all aggregates and $A$ is the total size of the concrete specimen.

Regarding the simulation of the aggregate spatial distribution, the random sampling principle of Monte Carlo's simulation method is used. This method is commonly called the take-and-place method. The random principle is applied by taking the aggregate sizes from a grading curve and placing each particle in the mortar matrix randomly so that intersection between aggregate is avoided. This method has been used by most researchers including Bazant et al. [14], Schlangen and Van Mier [15]. A different method can be used such as: the divide and fill method [16], the random particle drop method [17].

\section{APPLICATION}

The main purpose of this numerical example to demonstrate the potential of the phase field method to simulate the crack propagation in highly complex microstructure of concrete. For this purpose, the tensile test in [18] is numerically analyzed and the results are compared with the experimental one.

Fig. 2 shows the geometry and boundary conditions for uniaxial tests. It consists of $50 \mathrm{~mm} \times 50 \mathrm{~mm}$ square numerical specimens. The model is fixed at the bottom boundary and is subjected to a uniformly distributed displacement at the top boundary. In this study, the aggregate size distribution is generated by using the Fuller curve. $n$ is chosen equal to 0.5 . The aggregate particle whose size is greater than $2.36 \mathrm{~mm}$ is considered as coarse aggregate while fine aggregate together with cement matrix is treated as mortar. The interfacial transition zone between coarse aggregates and mortar matrix is neglected. Here, for the sake of simplicity, the coarse aggregate particles are geometrically represented by a circular shape.

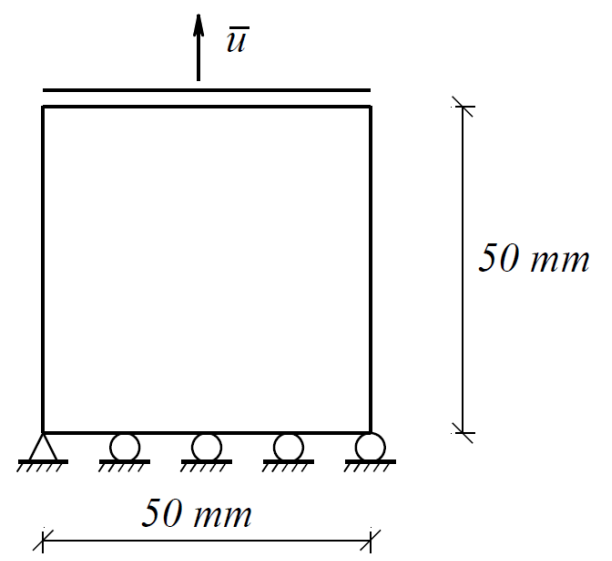

Fig. 2. Geometry of the specimen and boundary condition For normal strength concrete, the coarse aggre-

gate represents around $40-50 \%$ the concrete volume. In this study, the area fraction of coarse aggregate is assumed to be equal to $40 \%$. Coarse aggregates and mortar are described by linear elastic behavior. Similar material properties as in [12] are used in this study. Young's modulus is $70000 \mathrm{MPa}$ for coarse aggregates and is $25000 \mathrm{MPa}$ for mortar. Poisson's ratio of both coarse aggregates and mortar is 0.2. Fracture energy is $g_{c}=0.06 \mathrm{~N} / \mathrm{mm}$ for coarse aggregates and is $g_{c}=0.05 \mathrm{~N} / \mathrm{mm}$ for mortar.

The analyses are performed in plane stress condition and the out of plane thickness was unit. All analyses is ended at a displacement $0.08 \mathrm{~mm}$. The computation is performed with monotonic displacement increments of $\bar{u}=10^{-4} \mathrm{~mm}$. during 800 load 
increments. The length scale parameter is chosen as $l=0.35 \mathrm{~mm}$. In this study, the domain does not contain pre-existing cracks, we can not predict the crack nucleation and the crack pattern. Thus, in order to detect the crack nucleation, the domain is meshed by a regular triangular grid element whose characteristic size is about $h \approx 0.15 \mathrm{~mm}$. The total number of element is 249560. In [19], it showed that an element size $h<l / 2$ is needed in order to resolve the regularized crack surface $\Gamma_{l}(d)$, such that we have $\Gamma_{l}(d) \approx \Gamma$ in the finite element approximation.

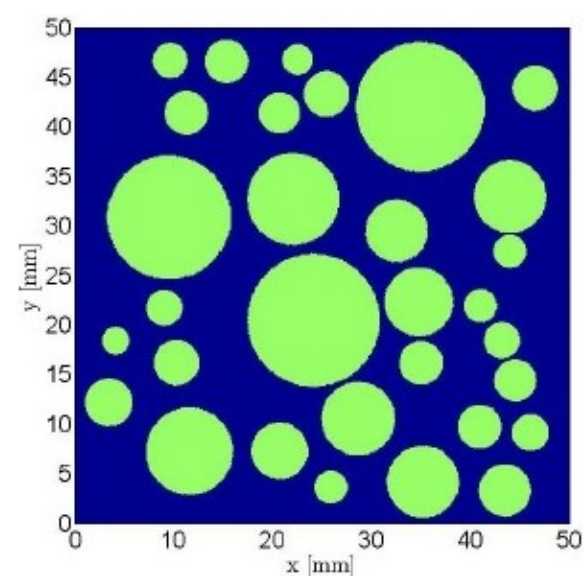

(a) Concrete sample 1

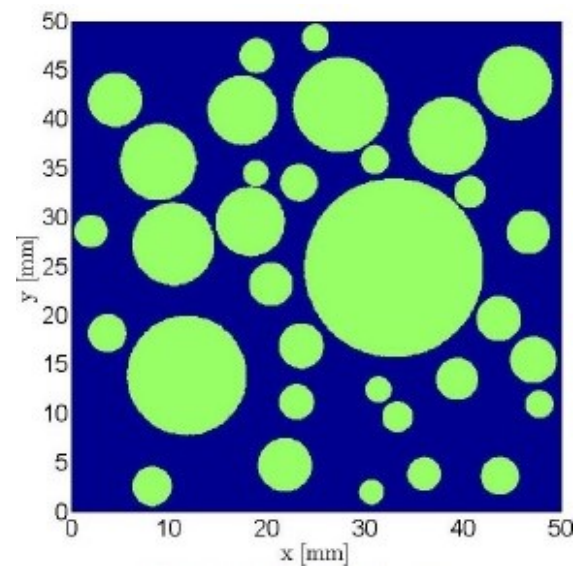

(c) Concrete sample 2

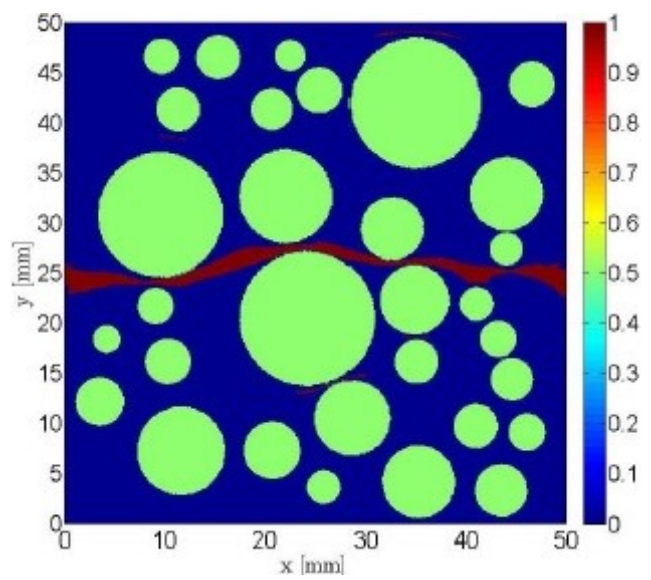

(b) Final crack pattern in concrete sample 1

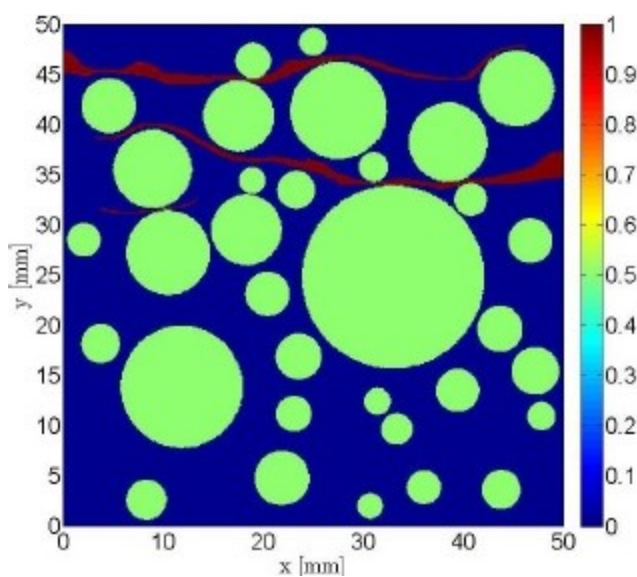

(d) Final crack pattern in concrete sample 2

Fig. 3. Predicted final crack pattern with different aggregate distribution (sample 1 and sample 2)

Figs. 3(a), 3(c) represent two random generations of aggregates, called concrete sample 1 and concrete sample 2. The results obtained in terms of final crack patterns are depicted in Figs. 3(b), 3(d). The cracks are represented by red color. Their corresponding stress-displacement curves are plotted in Fig. 4 and are compared with the result 


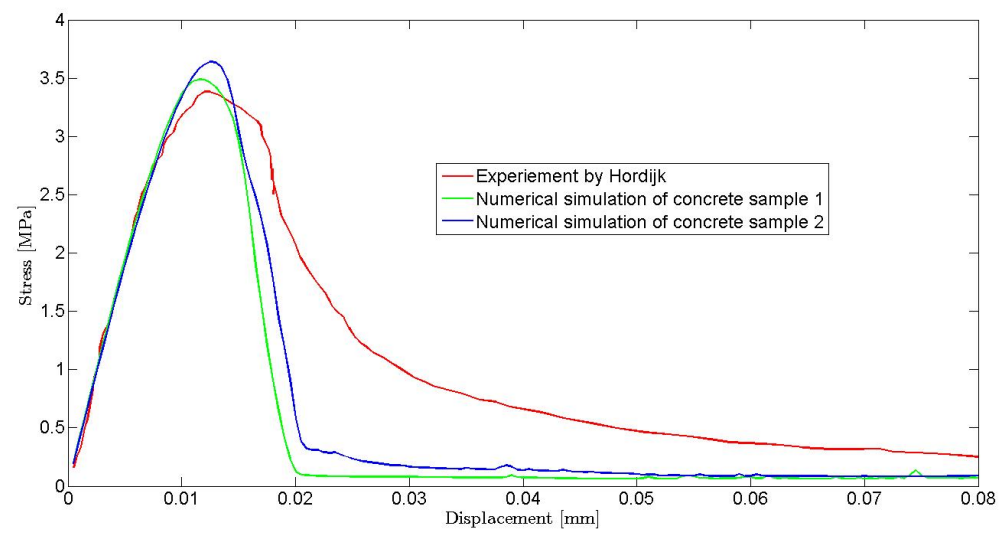

Fig. 4. Comparison of stress-displacement curves in uniaxial tension test

obtained experimentally by Hordijk [18]. It can be seen that the numerical and experimental results are in good agreement at the elastic stage. There is slightly difference in the peak stress. However, the numerical results underestimate the softening branch. It can be explained by the fact that some simplification are adopted in this study: the ITZ is neglected, the aggregate shape is represented by circular shape. The concrete sample fails either with one macrocracks (concrete sample 1) or with two crack (concrete sample 2). All macrocracks are predominantly perpendicular to the load direction. It can be seen that the post-peak stress obtained from concrete sample 1 drops more quickly than the one from concrete sample 2 . Thus, there is a lower disspated energy in concrete sample 1 . This behaviour may be attributed to smaller fracture area in single crack than two cracks.

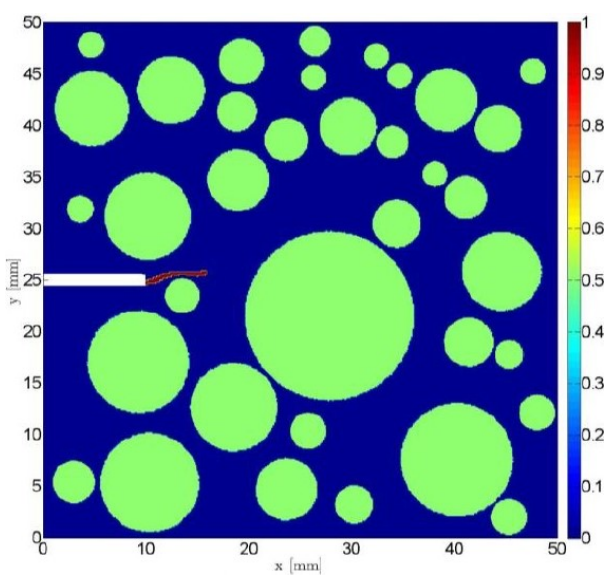

(a) Initial stage of crack propagation processes

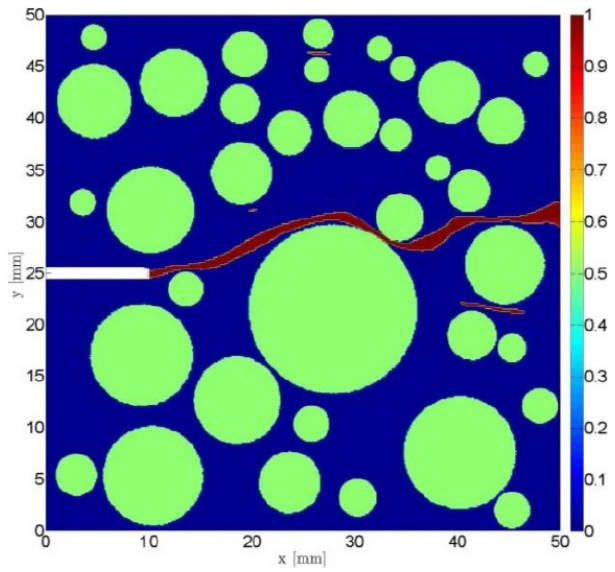

(b) Final stage of crack propagation processes

Fig. 5. Crack propagation processes in uniaxial tension test with pre-existing notch 
To futher verify the performance of the proposed model for fracture of concrete at mesoscale, a uniaxial test with pre-existing notch with the length of $20 \mathrm{~mm}$ and the depth of $1 \mathrm{~mm}$ is simulated. The same boundary condition, material properties in the aforementioned test is used. The crack propagation processes of concrete for pre-existing notch are shown in Fig. 5. At the initial stage, the crack are started from the notch Fig. 5(a). The crack orientation is consistent with the crack propagation orientation of mode I fracture. In the subsequent propagation processes, relatively large aggregates encountered and consequently, the crack path is changed Fig. 5(b). The crack runs around than propagating through aggregates, which is in good agreement with the realistic behaviour of normal concrete.

\section{CONCLUSION}

In this paper, we deal with the traditional "but complex" problem of modeling the damage and fracture behavior of concrete material. To do this, we construct an numerical approximation based on the phase field thermodynamic framework. Then, we are interested in only simple numerical tests composed of 2D configuration, circular aggregate, and no ITZ phase. In spite of this simplicity, some numerical results show good agreement with experimental observations, this is an interesting one and permit us to investigate this approach for further applications concerning the complex micro-structure of cement-based composite material.

\section{ACKNOWLEDGMENT}

This research is supported by Ministry of Education and Training under the grant number B2020-GHA-07.

\section{REFERENCES}

[1] G. Constantinides and F.-J. Ulm. The effect of two types of CSH on the elasticity of cementbased materials: Results from nanoindentation and micromechanical modeling. Cement and Concrete Research, 34, (1), (2004), pp. 67-80. https://doi.org/10.1016/s0008-8846(03)00230-8.

[2] P. Wriggers and S. O. Moftah. Mesoscale models for concrete: Homogenisation and damage behaviour. Finite Elements in Analysis and Design, 42, (7), (2006), pp. 623-636. https://doi.org/10.1016/j.finel.2005.11.008.

[3] N. Ile, X.-H. Nguyen, P. Kotronis, J. Mazars, and J. M. Reynouard. Shaking table tests of lightly rc walls: Numerical simulations. Journal of Earthquake Engineering, 12, (6), (2008), pp. 849-878. https://doi.org/10.1080/13632460801890430.

[4] T. T. Nguyen, J. Yvonnet, Q. Z. Zhu, M. Bornert, and C. Chateau. A phase field method to simulate crack nucleation and propagation in strongly heterogeneous materials from direct imaging of their microstructure. Engineering Fracture Mechanics, 139, (2015), pp. 18-39. https://doi.org/10.1016/j.engfracmech.2015.03.045.

[5] G. A. Francfort and J. J. Marigo. Revisiting brittle fracture as an energy minimization problem. Journal of the Mechanics and Physics of Solids, 46, (8), (1998), pp. 1319-1342. https://doi.org/10.1016/s0022-5096(98)00034-9.

[6] X. Li, D. Chu, Y. Gao, and Z. Liu. Numerical study on crack propagation in linear elastic multiphase composite materials using phase field method. Engineering Computations, 36, (1), (2019), pp. 307-333. https://doi.org/10.1108/EC-03-2018-0116. 
[7] C. Miehe, M. Hofacker, and F. Welschinger. A phase field model for rate-independent crack propagation: Robust algorithmic implementation based on operator splits. Computer Methods in Applied Mechanics and Engineering, (199), (2010), pp. 2765-2778. https://doi.org/10.1016/j.cma.2010.04.011.

[8] T. T. Nguyen, Y. Yvonnet, M. Bornert, C. C. Chateau, K. Sab, R. Romani, and B. Le Roy. On the choice of parameters in the phase field method for simulating crack initiation with experimental validation. International Journal of Fracture, 197, (2), (2016), pp. 213-226. https://doi.org/10.1007/s10704-016-0082-1.

[9] H. Amor, J. J. Marigo, and C. Maurini. Regularized formulation of the variational brittle fracture with unilateral contact: numerical experiments. Journal of the Mechanics and Physics of Solids, 57, (8), (2009), pp. 1209-1229. https://doi.org/10.1016/j.jmps.2009.04.011.

[10] T. T. Nguyen, J. Yvonnet, Q.-Z. Zhu, M. Bornert, and C. Chateau. A phase field method for computational modeling of interfacial damage interacting with crack propagation in realistic microstructures obtained by microtomography. Computer Methods in Applied Mechanics and Engineering, 312, (2016), pp. 567-595. https://doi.org/10.1016/j.cma.2015.10.007.

[11] T. T. Nguyen, J. Yvonnet, D. Waldmann, and Q. C. He. Phase field modeling of interfacial damage in heterogeneous media with stiff and soft interphases. Engineering Fracture Mechanics, (2019), pp. 106-547. https://doi.org/10.1016/j.engfracmech.2019.106574.

[12] C. M. López, I. Carol, and A. Aguado. Meso-structural study of concrete fracture using interface element I: numerical model and tensile behavior. Materials and Structures, 41, (2007), pp. 583-599. https://doi.org/10.1617/s11527-007-9314-1.

[13] X. F. Wang, Z. J. Yang, J. R. Yates, A. P. Jivkov, and C. Zhang. Monte carlo simulation of mesoscale fracture modelling of concrete with random aggregates and pores. Construction and Building Materials, 15, (2015), pp. 35-45. https://doi.org/10.1016/j.conbuildmat.2014.09.069.

[14] Z. P. Bazant, M. R. Tabbara, M. T. Kazemi, and G. Pijaudier-Cabot. Random particle model for facture of aggregate or fiber composites. Journal of Engineering Mechanic, 116, (1990), pp. 16861705. https://doi.org/10.1061/(asce)0733-9399(1990)116:8(1686).

[15] E. Schlangen and J. G. M. van Mier. Simple lattice model for numerical simulation of fracture of concrete materials and structures. Materials and Structures, 25, (156), (1992), pp. 534-542. https://doi.org/10.1007/bf02472449.

[16] G. D. Schutter and L. Taerwe. Random particle model for concrete based on Delaunay triangulation. Journal of Engineering Mechanic, 26, (156), (1993), pp. 1686-1705. https://doi.org/10.1007/bf02472853.

[17] J. G. M. van Mier and M. R. A. V. Vliet. Influence of microstructure of concrete on size/scale effects in tensile fracture. Engineering Fracture Mechanics, 70, (16), (2003), pp. 2281-2306. https://doi.org/10.1016/s0013-7944(02)00222-9.

[18] D. A. Hordijk. Tensile and tensile fatigue behaviour of concrete: experiments, modelling and analyses. Heron, 37, (1992), pp. 1-79.

[19] C. Miehe, M. Hofacker, and F. Welschinger. Thermodynamically consistent phasefield models of fractures: variational principles and multi-field FE implementations. International Journal for Numerical Method in Engineering, (83), (2010), pp. 1273-1311. https://doi.org/10.1002/nme.2861. 